\title{
Assessing cumulative dose distributions in combined external beam radiotherapy and intracavitary brachytherapy for cervical cancer by treatment planning based on deformable image registration
}

\author{
Jing Zeng ${ }^{1,2 \#}$, Jie Chen ${ }^{1 \#}$, Daguang Zhang ${ }^{1}$, Maobin Meng ${ }^{1}$, Bailin Zhang ${ }^{1}$, Pengpeng Qu ${ }^{2}$, Qingsong Pang ${ }^{1}$, \\ Ping Wang ${ }^{1}$ \\ ${ }^{1}$ Tianjin Medical University Cancer Institute and Hospital, National Clinical Research Centre for Cancer, Key Laboratory of Cancer Prevention \\ and Therapy, Tianjin's Clinical Research Center for Cancer, Tianjin, China; ${ }^{2}$ Department of Gynecologic Oncology, Tianjin Central Hospital of \\ Gynecology Obstetrics, Affiliated Hospital of Nankai University, Tianjin, China \\ Contributions: (I) Conception and design: J Zeng, M Meng; (II) Administrative support: P Wang, P Qu; (III) Provision of study materials or patients: \\ J Chen, B Zhang; (IV) Collection and assembly of data: D Zhang; (V) Data analysis and interpretation: Q Pang; (VI) Manuscript writing: All authors; \\ (VII) Final approval of manuscript: All authors. \\ \#These authors contributed equally to this work. \\ Correspondence to: Qingsong Pang. Tianjin Medical University Cancer Institute and Hospital Huanhuxi Road, Tianjin 300060, China. \\ Email: pangqingsong2013@163.com.
}

Background: This study aimed to validate the feasibility of deformable image registration (DIR) in assessing the cumulative dose distributions in combined external beam radiotherapy (EBRT) and intracavitary brachytherapy (ICBT) for cervical cancer.

Methods: This retrospective study included 23 patients with stage IIB disease treated with combined EBRT to the whole pelvis (50.4 Gy in 28 fractions) using an intensity-modulated radiotherapy technique with 6-MV X-ray, followed by three-dimensional (3D) ICBT (28 Gy in 4 fractions). Tumor gross target volume at diagnosis (GTV-Tinit), tumor gross target volume before brachytherapy, high-risk clinical target volume (HR-CTV), intermediate-risk clinical target volume (IR-CTV), and parametrium and organs at risk were recontoured on computed tomography images of EBRT and ICBT, respectively. The dose-volume parameters were also determined. The DIR results were reviewed using MIM Maestro (Reg Review) and modified by function (Reg Refine). To evaluate the accuracy of DIR, DIR-based cumulative dose-volume histogram (DVH) parameters and simple DVH parameter addition were compared using Wilcoxon rank-sum tests.

Results: The cumulative dose distributions of EBRT and four ICBT sessions were successfully illustrated using DIR. The mean tumor diameters were $68.35 \mathrm{~cm}^{3}$ at diagnosis and $29.63 \mathrm{~cm}^{3}$ at ICBT initiation. The mean tumor regression was $56.6 \%$. The median minimum dose covering $90 \%$ (D90) of HR-CTV, GTV-Tinit, IR-CTV, and parametrium were $69.58 \pm 4.94,68.81 \pm 7.98,59.28 \pm 3.78$, and $60.97 \pm 1.1 \mathrm{~Gy}_{\alpha / \beta}=10$, respectively, for DIR and $69.11 \pm 5.68,68.49 \pm 8.62,58.89 \pm 3.59$, and $61 \pm 1.49 \mathrm{~Gy}_{\alpha / \beta}=10$, respectively, with conventional simple DVH parameter addition.No statistically significant differences in dosimetric parameters were observed between the two methods.

Conclusions: Although there were limitations in the DIR accuracy, DIR-based dose accumulation was significantly beneficial in visually showing the cumulative dose distribution in the target area to clinicians in combined radiotherapy for cervical cancer in routine clinical practice.

Keywords: Cervical cancer; radiotherapy; deformable image registration (DIR); brachytherapy

Submitted Feb 25, 2020. Accepted for publication Aug 21, 2020.

doi: $10.21037 /$ tcr-20-1196

View this article at: http://dx.doi.org/10.21037/tcr-20-1196 


\section{Introduction}

The 2019 National Comprehensive Cancer Network (1) guidelines for Clinical Practice of Cervical Cancer 2019 (1st edition) recommend parametrial boosting in select cases with bulky parametrial/pelvic side wall disease after initial whole pelvic radiation, which has been added in the principle of radiotherapy. In such cases, the cumulative dose distributions of clinical targets should be determined and the dose-volume histograms (DVHs) calculated to identify the cumulative dose-volume associations for the target volumes and organs at risk (OARs) in combined external beam radiotherapy (EBRT) and intracavitary brachytherapy (ICBT). However, the accurate evaluation of cumulative dose distributions at the targets in the combined EBRT and ICBT is still a challenge for clinicians, specifically for patients with uncontrolled or recurrent tumors who require secondary radiotherapy.

Brachytherapy is an irreplaceable radiation treatment for cervical cancer. Recently, the field has transitioned from point- to volume-based planning (2), allowing for improved target volume and OAR visualization and enhanced dose conformality (3). When patients receive EBRT, brachytherapy is usually initiated toward the latter part of treatment. Sufficient primary tumor regression at this time permits satisfactory brachytherapy apparatus geometry. However, because of the different dose profiles and fractionations of ICBT, organ movement, and changes in target volume, the calculation of DVH parameters for the target volume and OARs may be difficult when assessing cumulative dose distributions with EBRT in conventional treatment planning systems. Several studies have determined the effect of deformable image registration (DIR) on cumulative OARs such as bladder and rectum DVH parameter summation for brachytherapy fractions (4-6). They demonstrated that several factors affect the systematic underestimation of dose. Specifically, the complete overlap of the DVH parameter hot spots may not always be accurate, and DIR in cervical cancer brachytherapy leads to lower average OAR DVH parameters $(5,6)$. To date, the clinical effect of DIR on cumulative OAR DVH parameter summation between brachytherapy fractions is also controversial. In combined EBRT and BT treatment, van Heerden $e t$ al. used structure-based DIR to evaluate cumulative DVH parameters for cervical cancer in the bladder and rectum (7). $\mathrm{Xu}$ et al. applied the full parameter addition methods with respect to the image registrationbased methods for evaluating combined EBRT and ICBT (8).
Their research proved that DIR could serve as an acceptable plan evaluation tool for clinical purposes in combined EBRT and BT treatment. In this study, we focused on the evaluation of cumulative dose distributions on targets and DVHs in combined radiotherapy for cervical cancer using a commercially available DIR algorithm (MIM Maestro ${ }^{\circledR}$ ) (9) in clinical practice. We present the following article in accordance with the STROBE reporting checklist (available at http://dx.doi.org/10.21037/tcr-20-1196).

\section{Methods}

\section{Patients and treatment}

Twenty-three patients having anteflexed or upright uterus with stage IIB disease previously treated with combined ICBT and EBRT were randomly selected for this retrospective study. All patients received EBRT to the whole pelvis including the parametrium (50.4 Gy in 28 fractions) using the intensity-modulated radiotherapy (IMRT) (Varian, IX, Pinnacle 9.8 plan system, USA) technique with 6-MV X-ray followed by three-dimensional (3D) ICBT (28 Gy in 4 fractions). Computed tomography (CT) [Philips, Brilliance BigBore (slice thickness, $5 \mathrm{~mm}$ )]-based 3D imageguided brachytherapy (Elekta, Nucletron microSelectron V3, Oncentra version 4.3 plan system, Sweden) was performed for the first fraction of ICBT. The other three fractions used the same initial CT-based brachytherapy planning. Since the ICBT and EBRT doses were delivered continuously in each fraction, their physical doses were summed directly, assuming the same biological effectiveness. The total equieffective prescription for a total point $\mathrm{A}$ dose was $90 \mathrm{~Gy}_{\alpha / \beta=10}$ (assuming an $\alpha / \beta$ ratio of $10 \mathrm{~Gy}$ ). ICBT was performed weekly for 4 consecutive weeks using high-dose rate brachytherapy. In all cases, tandem and ovoid with sufficient vaginal packing were used to ensure appropriate placement and planning. At least 6 Gy was prescribed to the high-risk clinical target volume (HR-CTV) minimum dose covering $90 \%$ of the tumor volume (D90) in each ICBT session. The dose constraint was $75 \mathrm{~Gy}_{\alpha / \beta=10}$ in the minimum doses to the highest irradiated $2 \mathrm{cc}$ area of the rectum (D2cc). The dose prescriptions and target coverages were modified based on the dose constraints for D2cc of $90 \mathrm{~Gy}_{\alpha / \beta=3}$ to the bladder and $70 \mathrm{~Gy}_{\alpha / \beta=3}$ to the rectum according to Groupe Européen de Curiethérapie and the European Society for Radiotherapy \& Oncology (GECESTRO) recommendations (10).

The study was conducted in accordance with the 
Table 1 Parametrial border definitions according to the CTstandardized contour guidelines (11)

\begin{tabular}{ll}
\hline Border & \multicolumn{1}{c}{ Structures } \\
\hline Superior & Fallopian tube or broad ligament (RTOG) \\
Inferior & Levator ani/pelvic floor muscles \\
Anterior & $\begin{array}{l}\text { Posterior bladder or posterior border of } \\
\text { external iliac vessels }\end{array}$ \\
Posterior & Mesorectal fascia and uterosacral ligaments \\
Lateral & Lateral pelvic sidewall; medial internal \\
& obturator/piriformis muscle/ischial ramus \\
Medial & Cervix \\
\hline
\end{tabular}

RTOG, Radiation Therapy Oncology Group.

Declaration of Helsinki (as revised in 2013). The study was approved by the Human Investigation Committee of Tianjin Medical University Cancer Institute and Hospital, Tianjin, China (No. bc2019029). Written informed consent for the scientific use of the clinical data was obtained from all patients.

\section{Computed tomography simulation}

This study requested that patients collect urine 1 hour before undergoing CT imaging for EBRT treatment planning to maintain the relative horizontal position of the uterus. We used the initial planning CT image with a $5-\mathrm{mm}$ slice thickness per patient rather than per fraction.

\section{Target and organ-at-risk definitions}

The CTV of EBRT comprised the cervical tumor gross target volume at diagnosis (GTV-Tinit), the entire uterus, the parametrium, at least the upper half of the vagina, and the pelvic lymph nodes (including the common, external, and internal iliac and presacral lymph nodes). The planned target volume of EBRT included the CTV plus a 7-mm safety margin. For ICBT, CT contour of the HR-CTV, intermediate-risk CTV (IR-CTV), cervical tumor gross target volume before brachytherapy, parametrium, rectum, and bladder were adapted based on the CT-standardized contour guidelines (11). Based on the findings of gynecologic examinations at diagnosis and brachytherapy, the HR-CTV was delineated. Next, the IR-CTVs were generated by adding a $1-\mathrm{cm}$ expansion around the HRCTV, modifying the disease extent at diagnosis with a comparison to the initial extension in either the clinical diagram or initial magnetic resonance imaging (MRI) scan, and modifying the volume by deleting contours extending into the bladder, sigmoid, or rectum. Table 1 shows the details of the parametrial borders.

\section{Image registration}

Image registration was performed using MIM Maestro version 6.7.10 (MIM Software Inc., Cleveland, OH, USA) (12). CT image datasets from ICBT were rigidly fused by matching structures on CT images from IMRT. The rigid registration was determined to be "as good as possible" based on bone alignment. DIR was performed to accumulate doses of four fractions of ICBT with the same dose of the initial planning CT image per fraction. The results of the DIR were carefully reviewed using the MIM Maestro function in Reg Review and modified using the Reg Refine function. Figure 1 shows the schematics and workflow. Patients were required to defecate before being treated to avoid gas artifacts. Rectal dilatation was confirmed by treatment planning CT of EBRT and ICBT. If gas is found in the rectum during ICBT, gas drainage is performed to reduce rectal expansion. Subsequently, CT is performed again.

\section{Dose-volume evaluations}

The radiotherapy doses were converted into biologically equivalent doses in $\mathrm{Gy}_{\mathrm{EQD} 2}$ using the linear quadratic model with $\alpha / \beta=10$ Gy for tumor tissues and 3 Gy for normal tissues (13). To evaluate the combination of IMRT and ICBT for each plan generated, we calculated the median value of all plans based on the dose values received by $90 \%$ of the target volume (D90), the dose received by $100 \%$ of the target volume (D100), and the volume receiving 100\% of the prescription dose (V100) compared to those for conventional simple DVH parameter addition, according to the EMBRACE II study (14) (Table 2). "Simple straight addition" was performed with the rigid image registration based on straight addition where there will be one final D90.

\section{Statistical analyses}

Wilcoxon rank-sum tests were used to investigate the differences between DIR-based dose accumulation and DVH parameter addition with regard to D90, D100, and 

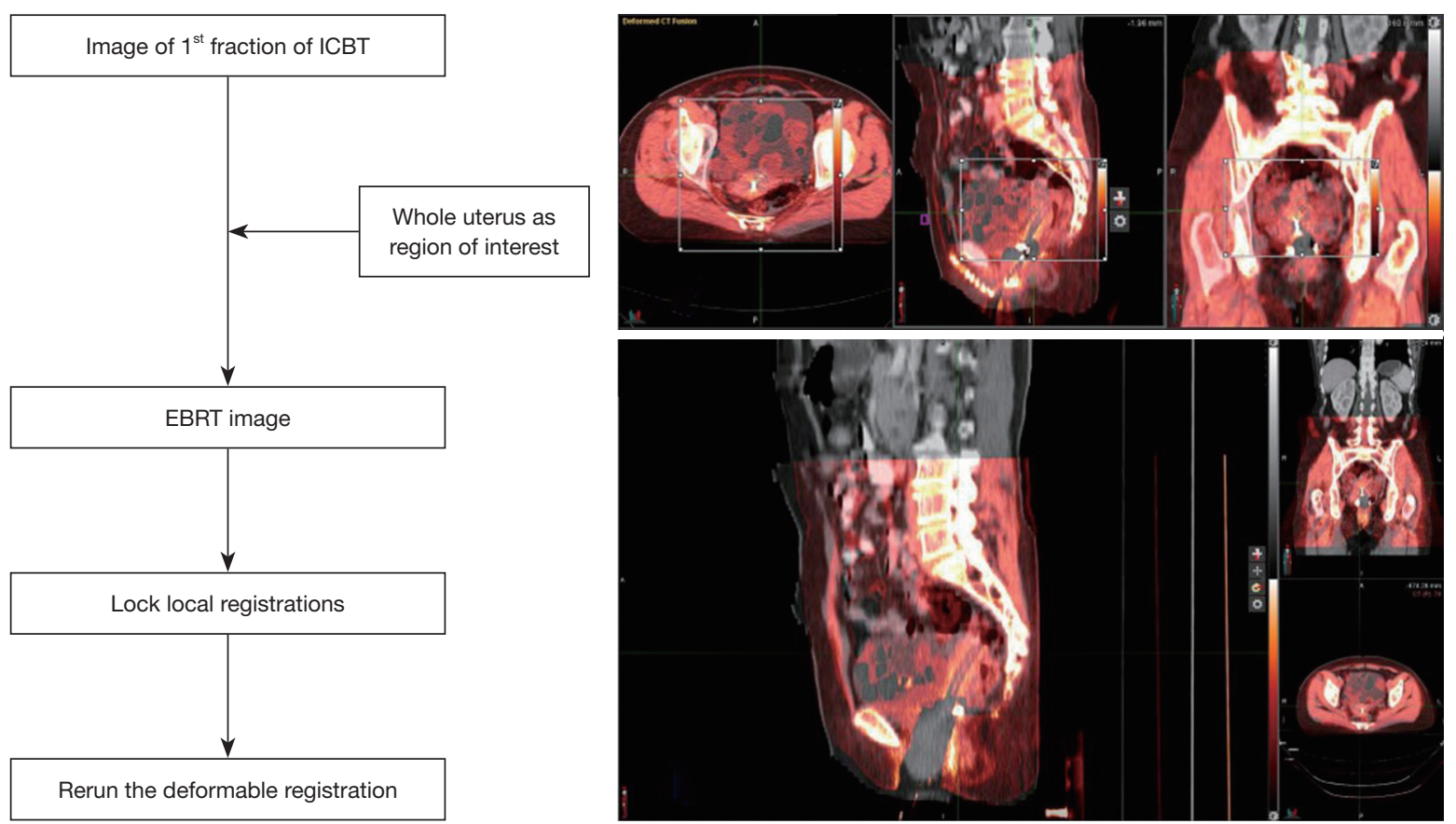

Figure 1 Reg refine-based correction of deformation.

Table 2 Median D100, D90, and V100 values of the GTV-Tinit, HR-CTV, IR-CTV, and parametrium $(\alpha / \beta=10$ Gy)

\begin{tabular}{|c|c|c|c|}
\hline Parameter & DIR & Simple addition & $P$ value \\
\hline \multicolumn{4}{|l|}{ GTV-Tinit } \\
\hline D100 (Gy) & $61.89 \pm 3.53$ & $61.55 \pm 4.15$ & 0.330 \\
\hline D90 (Gy) & $68.81 \pm 7.98$ & $68.49 \pm 8.62$ & 0.301 \\
\hline V100 (\%) & $30.27 \pm 4.44$ & $30.03 \pm 4.61$ & 0.057 \\
\hline D100 (Gy) & $60.45 \pm 3.79$ & $60.25 \pm 3.71$ & 0.144 \\
\hline D90 (Gy) & $69.58 \pm 4.94$ & $69.11 \pm 5.68$ & 0.52 \\
\hline V100 (\%) & $35.56 \pm 4.96$ & $35.28 \pm 4.91$ & 0.117 \\
\hline \multicolumn{4}{|l|}{ IR-CTV } \\
\hline V100 (\%) & $28.92 \pm 6.13$ & $28.92 \pm 6.13$ & 0.616 \\
\hline \multicolumn{4}{|l|}{ Parametrium } \\
\hline D100 (Gy) & $55.71 \pm 2.73$ & $55.51 \pm 2.27$ & 0.692 \\
\hline D90 (Gy) & $60.97 \pm 1.1$ & $61 \pm 1.49$ & 0.648 \\
\hline
\end{tabular}

GTV-Tinit, GTV at diagnosis; HR-CTV, high-risk clinical target volume; IR-CTV, intermediate-risk clinical target volume; V100, volume receiving $100 \%$ of the prescription dose; D90, dose received by $90 \%$ of the target volume; D100, dose received by $100 \%$ of the target volume. 


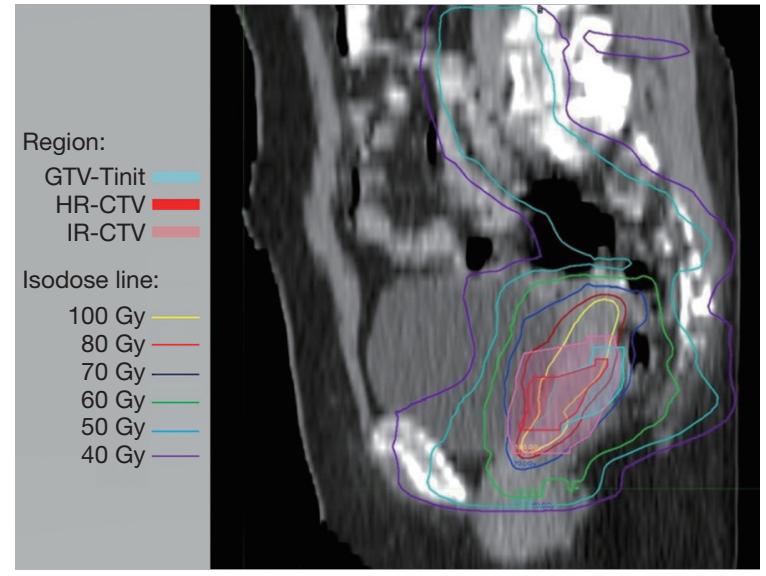

Figure 2 Total dose $\left(\mathrm{Gy}_{\alpha / \beta=10}\right)$ distributions for the combined external beam radiotherapy and intracavitary brachytherapy (EBRT+ ICBT) plan. EBRT, external beam radiotherapy; ICBT, intracavitary brachytherapy.

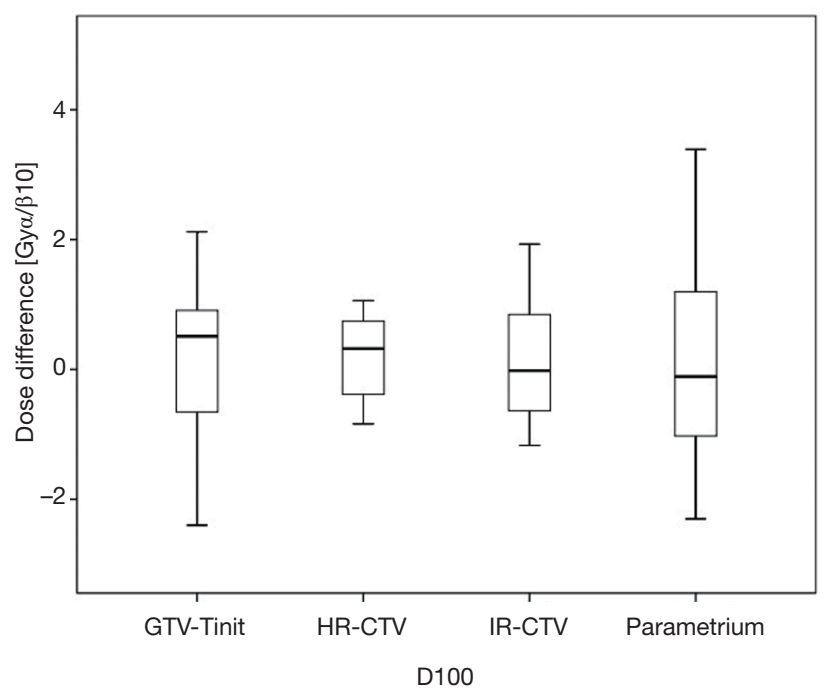

Figure 3 Dose received by $100 \%$ of the target volume (D100) differences between DVH parameters obtained from DVH parameter addition and from the averaged DIR-based dose accumulations. D100, dose received by $100 \%$ of the target volume; DVH, dose-volume histogram; DIR, deformable image registration.

V100 deviations for GTV-Tinit, HR-CTV, IR-CTV, and parametrium, respectively. Wilcoxon signed-rank tests were performed for each of the parameters mentioned above to disclose systematic differences between DIRbased accumulation and DVH parameter addition. Analyses

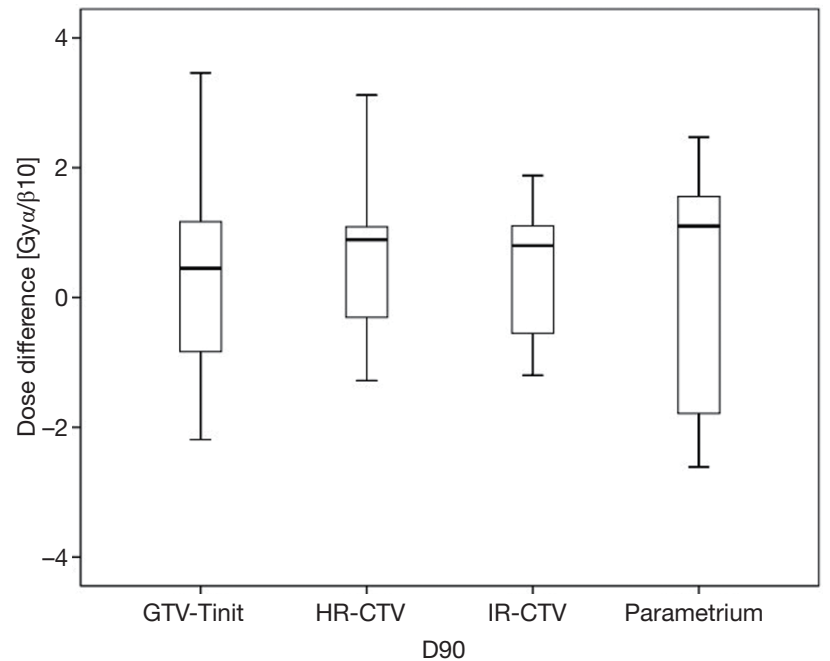

Figure 4 Dose received by $90 \%$ of the target volume (D90) differences between DVH parameters obtained from DVH parameter addition and from the averaged DIR-based dose accumulations. D90, dose values received by $90 \%$; DVH, dosevolume histogram.

were performed using the International Business Machines Corporation Statistical Package for the Social Sciences Statistics for Windows version 19.0.

\section{Results}

\section{Cumulative doses}

The cumulative dose distributions, comprising EBRT and four sessions of ICBT, were successfully illustrated using DIR (Figure 2). The HR-CTV was covered with $70-80 \mathrm{~Gy}_{\alpha / \beta=10}$. The parametria were covered with $50-60 \mathrm{~Gy}_{\alpha / \beta=10}$.

\section{Gross target volume and DVH parameters}

Among the 23 patients included in the present study, the mean tumor diameters were $68.35 \mathrm{~cm}^{3}$ at diagnosis and $29.63 \mathrm{~cm}^{3}$ at ICBT initiation. The mean tumor regression was $56.6 \%$. The mean \pm standard deviation (SD) HRCTV D90 and D100 of the GTV-Tinit, IR-CTV, and parametrium were $69.58 \pm 4.94,61.89 \pm 3.53,54.16 \pm 4.01$, and $55.71 \pm 2.73 \mathrm{~Gy}_{\alpha / \beta=10}$, respectively, for DIR and $69.11 \pm 5.68$, $61.55 \pm 4.15,53.99 \pm 3.93$, and $55.51 \pm 2.27 \mathrm{~Gy}_{\alpha / \beta=10}$, respectively, for the conventional simple DVH parameter addition (Figures 3-5). No statistically significant differences were observed in the dosimetric parameters between the 


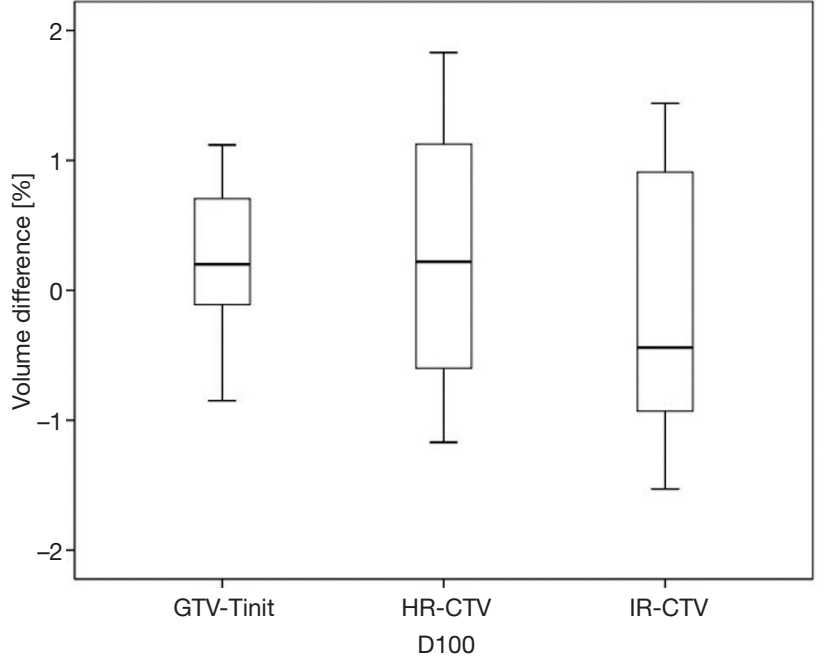

Figure 5 Volume receiving $100 \%$ of the prescription dose (V100) differences between DVH parameters obtained from DVH parameter addition and from the averaged DIR-based dose accumulations. V100\%, volume receiving $100 \%$ of the prescription dose; DVH, dose-volume histogram; DIR, deformable image registration.

two calculation methods (Table 2). No systematic differences were observed between DIR-based accumulation and DVH parameter addition $(\mathrm{P}=0.11)$.

\section{Discussion}

DIR is now accepted as a plan evaluation tool for clinical purposes at several radiation oncology centers not only for automatic contouring but also for diagnostic image registration and dose accumulation. The quality of DIR is dependent on image quality, user skills, and software algorithm. Several centers also use an Excel sheet-based EQD2 dose composite recommended by the American Brachytherapy Society. In these applications, these registrations must be characterized in phantom studies and also checked on a per-patient basis around regions in which deformable registration guided the clinical decisions (12).

DIR accuracy is of utmost importance in evaluating the DIR-based cumulative dose distributions and DVHs of the tumor and OARs. All selected cases in the present study had anteflexed or upright uterus. The uterus became horizontal after the insertion of the uterine tube in the ICBT. Considering the comfort of patients without general anesthesia in every ICBT session, the patients were only required to collect urine during ICBT to stabilize bladder size and position. With these methods, the cumulative images by rigid fusion were based on the bony structure (Figure 2).

However, in brachytherapy, the target volume and parts of the OARs could move together with the applicator after the applicator's implant (15). As the applicator changed its position relative to the bony structures, it is essential to match the region of interest, including the target volume and the parts of the OARs moving together with the applicator rather than only matching the brachytherapy applicator or bony structures (16). Therefore, the use of a proper deformation registration method based on a rigid registration improved fusion accuracy with automatic tools in planning systems (17).

Contouring and reconstruction performed in the same image series allowed the dose to targets and organs to be directly calculated without any image fusion. However, the fusion uncertainties could translate into absorbed dose calculation when several image series were combined. For example, contouring was performed in one image series and reconstruction in another. Tanderup et al. reported that uncertainties resulted in a miscalculation of DVH dose parameters by typically $4 \%$ to $6 \% \mathrm{~mm}^{-1}$ of fusion error between CT and MRI (18). van Heerden et al. (19) recently used DIR to determine the absorbed dose distribution of daily image-guided adaptive EBRT and brachytherapy. The cone-beam CT of the first EBRT fraction and the brachytherapy planning MRI were registered by DIR. The cumulative dose to $2 \mathrm{~cm}^{3}$ with the highest dose (D $2 \mathrm{~cm}^{3}$ ) from EBRT and brachytherapy to the bladder and rectum were calculated and compared to those for direct addition, assuming a uniform EBRT dose (UD). The differences between UD and DIR were less than 3.9 $\mathrm{Gy}_{\mathrm{EQD} 2}$. Our study only used CT images with the same field of view and slice thickness for fusion. The DIR-based DVH parameter values did not differ significantly from those derived from the conventional simple DVH parameter addition (Figure 3). According to the Groupe Européen de Curiethérapie and the GEC-ESTRO recommendations (10), the conventional simple DVH parameters for the target volumes and OARs in EBRT and each ICBT session were simply added to estimate the cumulative DVH parameters.

Mayr et al. reported that the tumor could shrink from $60 \%$ to $80 \%$ of the pre-therapeutic tumor volume during the first 2-3 weeks of EBRT (20). The patient's anatomy at the time of brachytherapy with the applicator in situ differed significantly from that at EBRT. In this study, the mean tumor regression was $56.6 \%$ when brachytherapy 
was initiated after 3-4 weeks of EBRT. Although pelvic organs, such as the bladder, rectum, and intestine, differed significantly in movement and filling, the processed image fusion met the requirements for clinical target dose assessment by planning systems.

The previous analyses proved that ICBT, with the new target concept based on HR-CTV and IR-CTV and combined with the pear concept used in the past for ICBT, had a significantly high local control rate (21-23). Thus, it was appropriate and feasible to evaluate the extension of the dose into the parametria with cervical cancer $(24,25)$. Nomden et al. compared the treatment planning of four cervical cancer patients with different sizes of HR-CTV between four centers (23). In their study, the median HRCTV D90 was 68.9 Gy, whereas the mean \pm SD HR-CTV D90 of our result was $69.58 \pm 4.94 \mathrm{~Gy}_{\alpha / \beta=10}$ in the treatment plan with equieffective prescription for a total point A dose of $90 \mathrm{~Gy}_{\alpha / \beta=10}$. The results did not meet the demand for HRCTV $\geq 80 \mathrm{~Gy}_{\alpha / \beta=10}$ for large or poorly responding tumors (1), for a total point A prescription dose (as recommended in the guidelines) of $80 \mathrm{~Gy}_{\alpha / \beta=10}$. The pear-shaped isodose without optimization implied a large non-conformality region compared to the HR-CTV definition. Although the doses to the HR-CTVs and OARs could be regulated through optimization, large residual or asymmetrically located tumors could not be managed by ICBT alone (26). A satisfactory D90 was not achieved for extensive tumors with an optimized ICBT approach alone because the main role of optimization was to reduce the D2cc to the OARs (27). Thus, clinicians must always consider the risk of local recurrence due to insufficient doses caused by these techniques. Furthermore, adequate evaluation of ICBT with EBRT is required for further parametrial-boosting radiotherapy.

According to the GEC-ESTRO recommendations (10), IR-CTV included the GTVres and residual pathological tissue. Margins were added for suspected adjacent residual microscopic disease in the direction of potential residual microscopic spread considering the natural anatomical borders. The selection and contouring of the IR-CTV were based on macroscopic tumor extension at diagnosis (GTV-Tinit), which had a high risk of local recurrence due to insufficient doses. In clinical practice, the initial extension, including both the clinical diagram and initial MRI scan, is crucial for IR-CTV. The parauterine tissue was the first site of cervical cancer invasion and had a high risk of local recurrence of cervical cancer. A lack of parametrial invasion was also a decisive factor for surgical treatment. Therefore, the assessment of cumulative dose distributions in the parauterine tissue was considered in the clinical setting. In this study, the mean \pm SD D90 of the IR-CTV and parametria were $54.16 \pm 4.01$ and $55.71 \pm 2.73 \mathrm{~Gy}_{\alpha / \beta=10}$, respectively, which were comparable to the clinical prescription dose.

However, we did not successfully evaluate the dosage of OARs due to their large anatomical changes after deformation registration for the following reasons. First, we did not strictly control the bladder volume during preimaging preparations for EBRT and ICBT treatment. Thus, the target volume of the bladder changed. Second, the intestinal position and volume could vary during the 2 weeks of treatment. Additionally, the 5 -mm CT slice thickness used in our study could result in high calculation and fusion uncertainty, which is a main limitation of our study. Furthermore, we fused images and produced cumulative dose distributions only for the first ICBT imaging instead of the other four fractions using DIR. During fractionated brachytherapy, the tumors might diminish further. The position and volume of the rectum and bladder could vary despite pre-imaging preparations. Related studies (11) reported that pre-imaging preparation did not prevent changes in HR-CTV volume. These positional and volumetric changes could influence the accuracy of image fusion in DIR.

\section{Conclusions}

Although it showed no significance in all DVH parameters between DIR and straight addition, DIR-based dose accumulation was significantly helpful to visually show the cumulative dose distribution in the target area to clinicians in combined radiotherapy for cervical cancer in routine clinical settings.

\section{Acknowledgments}

Funding: None.

\section{Footnote}

Reporting Checklist: The authors have completed the STROBE reporting checklist. Available at http://dx.doi. org/10.21037/tcr-20-1196

Data Sharing Statement: Available at http://dx.doi. org/10.21037/tcr-20-1196 
Peer Review File: Available at http://dx.doi.org/10.21037/tcr20-1196

Conflicts of Interest: All authors have completed the ICMJE uniform disclosure form (available at http://dx.doi. org/10.21037/tcr-20-1196). The authors have no conflicts of interest to declare.

Ethical Statement: The authors are accountable for all aspects of the work in ensuring that questions related to the accuracy or integrity of any part of the work are appropriately investigated and resolved. The study was conducted in accordance with the Declaration of Helsinki (as revised in 2013). The study was approved by institutional Ethics Review Board of Tianjin Medical University Cancer Institute and Hospital (No. bc2019029) and informed consent was taken from all the patients.

Open Access Statement: This is an Open Access article distributed in accordance with the Creative Commons Attribution-NonCommercial-NoDerivs 4.0 International License (CC BY-NC-ND 4.0), which permits the noncommercial replication and distribution of the article with the strict proviso that no changes or edits are made and the original work is properly cited (including links to both the formal publication through the relevant DOI and the license). See: https://creativecommons.org/licenses/by-nc-nd/4.0/.

\section{References}

1. Koh WJ, Abu-Rustum NR, Bean S, et al. Cervical Cancer, Version 3.2019, NCCN Clinical Practice Guidelines in Oncology. J Natl Compr Canc Netw 2019;17:64-84.

2. Kirisits C, Pötter R, Lang S, et al. Dose and volume parameters for MRI-based treatment planning in intracavitary brachytherapy for cervical cancer. Int J Radiat Oncol Biol Phys 2005;62:901-11.

3. Pötter R, Dimopoulos J, Georg P, et al. Clinical impact of MRI assisted dose volume adaptation and dose escalation in brachytherapy of locally advanced cervix cancer. Radiother Oncol 2007;83:148-55.

4. Flower E, Do V, Sykes J, et al. Deformable image registration for cervical cancer brachytherapy dose accumulation: Organ at risk dose-volume histogram parameter reproducibility and anatomic position stability. Brachytherapy 2017;16:387-92 .

5. Jamema SV, Mahantshetty U, Andersen E, et al. Uncertainties of deformable image registration for dose accumulation of high-dose regions in bladder and rectum in locally advanced cervical cancer. Brachytherapy 2015;14:953-962.

6. Flower EE, Do V, Sykes J, et al. Deformable Image Registration (DIR) for Cervical Cancer Brachytherapy: A Comparison of the Reproducibility of Three Different Methods and the Effects of DIR on the Anatomical Stability of OAR DVH Parameters. Brachytherapy 2016;15:s101.

7. van Heerden LE, Houweling AC, Koedooder K, et al. Potential Added Value of Structure-Based Deformable Image Registration for Dose Accumulation in External Beam Radiotherapy and Brachytherapy in Cervical Cancer. Brachytherapy 2016;15:s36.

8. Xu Z, Traughber BJ, Fredman E, et al. Appropriate Methodology for EBRT and HDR Intracavitary/ Interstitial Brachytherapy Dose Composite and Clinical Plan Evaluation for Patients With Cervical Cancer. Pract Radiat Oncol 2019;9:e559-71.

9. Piper J, Nelson A. Jason Harper MIM Software Inc. Deformable Image Registration in MIM Maestro ${ }^{\mathrm{TM}}$ Evaluation and Description. Available online: www. mimsoftware.com

10. Pötter R, Haie-Meder C, Van Limbergen E, et al. GEC ESTRO Working Group: Recommendations from gynaecological (GYN) GEC ESTRO working group (II): concepts and terms in 3D image-based treatment planning in cervix cancer brachytherapy - 3D dose volume parameters and aspects of $3 \mathrm{D}$ image-based anatomy, radiation physics, radiobiology. Radiother Oncol 2006;78:67-77.

11. Viswanathan AN, Dimopoulos J, Kirisits C, et al. Computed Tomography Versus Magnetic Resonance Imaging-Based Contouring in Cervical Cancer Brachytherapy: Results of a Prospective Trial and Preliminary Guidelines for Standardized Contours. Int J Radiat Oncol Biol Phys 2007;68:491-8.

12. Nie K, Pouliot J, Smith E, et al. Performance variations among clinically available deformable image registration tools in adaptive radiotherapy - how should we evaluate and interpret the result? J Appl Clin Med Phys 2016;17:328-40.

13. Dale RG. The application of the linear-quadratic doseeffect equation to fractionated and protracted radiotherapy. Br J Radiol 1985;58:515-28.

14. Pötter R, Tanderup K, Kirisits C, et al. The EMBRACE II study: The outcome and prospect of two decades of evolution within the GEC-ESTRO GYN working group and the EMBRACE studies. Clin Transl Radiat Oncol 2018;9:48-60. 
15. Gerbaulet A, Pötter R, Haie-Meder C. Cervix cancer. In: Gerbaulet A, Pötter R, Mazeron JJ, et al. editors. The GEC ESTRO handbook of brachytherapy. Brussels: ESTRO, 2002: 301-63.

16. Hellebust TP, Kirisits C, Berger D, et al. Recommendations from Gynaecological (GYN) GECESTRO Working Group: considerations and pitfalls in commissioning and applicator reconstruction in 3D imagebased treatment planning of cervix cancer brachytherapy. Radiother Oncol 2010;96:153-60.

17. Nesvacil N, Pötter R, Sturdza A, et al. Adaptive image guided brachytherapy for cervical cancer: a combined MRI-/CT-planning technique with MRI only at fifirst fraction. Radiother Oncol 2013;107:75-81.

18. Tanderup K, Hellebust TP, Lang S, et al. Consequences of random and systematic reconstruction uncertainties in $3 \mathrm{D}$ image-based brachytherapy in cervical cancer. Radiother Oncol 2008;89:156-63.

19. van Heerden LE, Visser J, Koedooder K, et al. Role of deformable image registration for delivered dose accumulation of adaptive external beam radiation therapy and brachytherapy in cervical cancer. J Contemp Brachytherapy 2018;10:542-50.

20. Mayr NA, Yuh WT, Taoka T, et al. Serial therapy-induced changes in tumor shape in cervical cancer and their impact on assessing tumor volume and treatment response. AJR Am J Roentgenol 2006;187:65-72.

21. Chargari C, Magne N, Dumas I, et al. Physics contributions and clinical outcome with 3D-MRI-based pulsed-dose-rate intracavitary brachytherapy in cervical cancer patients. Int J
Radiat Oncol Biol Phys 2009;74:133-9.

22. Lindegaard JC, Fokdal LU, Nielsen SK, et al. MRI-guided adaptive radiotherapy in locally advanced cervical cancer from a Nordic perspective. Acta Oncol 2013;52:1510-9.

23. Nomden CN, de Leeuw AA, Van Limbergen E, et al. Multicentre treatment planning study of MRI-guided brachytherapy for cervical cancer: comparison between tandem-ovoid applicator users. Radiother Oncol 2013;107:82-7.

24. Pötter R, Georg P, Dimopoulos JC, et al. Clinical outcome of protocol-based image (MRI) guided adaptive brachytherapy combined with $3 \mathrm{D}$ conformal radiotherapy with or without chemotherapy in patients with locally advanced cervical cancer. Radiother Oncol 2011;100:116-23.

25. Jürgenliemk-Schulz IM, Tersteeg RJ, Roesink JM, et al. MRI-guided treatment-planning optimisation in intracavitary or combined intracavitary/ interstitial PDR brachytherapy using tandem ovoid applicators in locally advanced cervical cancer. Radiother Oncol 2009;93:322-30.

26. Nishikawa R, Yoshida K, Ebina Y, et al. Comparison of dosimetric parameters in the treatment planning of magnetic resonance imaging-based intracavitary image-guided adaptive brachytherapy with and without optimization using the central shielding technique. J Radiat Res 2018;59:316-26.

27. Fokdal L, Sturdza A, Mazeron R, et al. Image guided adaptive brachytherapy with combined intracavitary and interstitial technique improves the therapeutic ratio in locally advanced cervical cancer: analysis from the retro EMBRACE study. Radiother Oncol 2016;120:434-40.
Cite this article as: Zeng J, Chen J, Zhang D, Meng M, Zhang B, Qu P, Pang Q, Wang P. Assessing cumulative dose distributions in combined external beam radiotherapy and intracavitary brachytherapy for cervical cancer by treatment planning based on deformable image registration. Transl Cancer Res 2020;9(10):6107-6115. doi: 10.21037/tcr-20-1196 